\title{
Prevalencia de riesgo de apnea obstructiva del sueño en población adulta chilena
}

\author{
JUAN CARRILLO A.*, CLAUDIO VARGAS R.**, \\ ARIEL CISTERNAS V.* y PEDRO OLIVARES-TIRADO***
}

\section{Prevalence of risk of obstructive sleep apnea in Chilean adult population}

Introduction: Obstructive sleep apnea (OSA) is a neglected chronic disease probably emerging under appropriate epidemiological conditions in Chile. Our goal was to estimate the prevalence of OSA risk in adult population. Patients and Methods: From the $2^{\text {nd }}$ Chilean Health Survey 2010 (NHS), we estimate the prevalence of risk of OSA in population $\geq 18$ years, as a derived proxy from STOPBang Questionnaire. A clinical prediction rule-CPR: habitual snoring, daytime sleepiness, nocturnal breathing pauses, blood hypertension, $B M I>35 \mathrm{~kg} / \mathrm{m}^{2}$, age $>50$ y.o., neck circumference $\geq 43 \mathrm{~cm}$ (men) and $\geq 41 \mathrm{~cm}$ (women) and male, was constructed. According to the total score subjects were classified as: Low (<3), Medium (3-4), and High Risk ( $\geq$ 5). SPSS Software (v22) modules for complex survey was used to obtain population prevalence and 95\% confidence intervals. Results: 5,069 records were obtained, mean age $48 \pm 18$ years, $60 \%$ women. A subsample of 4,234 fulfil the criteria per the CPR. The expanded sample (representing 11,279,865 persons), yielded the following results: Low risk $60.7 \%$ (CI 95\%, 58-63.4), Medium 31.1\% (28.7-33.6) and High 8.2\% (7-9.5). Men with Low risk 45.8\% (41.7-49.9), Medium 41.1\% (37.3-45.1), High 13.1\% (11-15.5). Women with Low risk 74.6\% (71.677.4), Medium 21.8\% (19.4-24.4), High 3.6\% (2.5-5.1). We observed an increasing trend in High risk of OSA from 0.3\% (0-1.8) in 18-24 years old group to a 22.9\% (18.4-28.2) in people aged $>65$ years old. Men with the highest prevalence of High risk OSA are in 7 of the 15 Regions: Araucania (24\%), Aysén (21.3\%), Coquimbo (18\%), Maule (17.8\%), Bio-Bio (17\%), Arica (16,2\%) and O'Higgins (15.7\%). Conclusion: OSA is a prevalent condition in Chilean Population, is higher in men than in women and a positive age trend of high risk OSA was observed.

Key words: Sleep apnea, obstructive; risk; prevalence; surveys and questionnaires.

\section{Resumen}

Introducción: En Chile la apnea obstructiva del sueño (AOS) es una enfermedad crónica insuficientemente reconocida, que probablemente emerge bajo condiciones epidemiológicas apropiadas. Nuestro objetivo fue estimar su prevalencia en nuestra población adulta. Pacientes y Métodos: A través de la $2^{a}$ Encuesta Nacional de Salud (ENS) 2010, estimamos la prevalencia de riesgo de AOS en población $\geq 18$ años de edad. Derivada del STOP-Bang Questionnaire construimos una regla de predicción clínica-RPC: ronquido habitual, somnolencia diurna, pausas respiratorias nocturnas, hipertensión arterial, IMC $>35 \mathrm{~kg} / \mathrm{m}^{2}$, edad $>50$ años, circunferencia cervical $\geq 43 \mathrm{~cm}$ (hombres) y $\geq 41 \mathrm{~cm}$ (mujeres), sexo: hombre. Según el puntaje total, el riesgo de los sujetos se clasificó como: Bajo $(<3)$, Medio (3-4) y Alto ( $\geq 5$ ). Para obtener prevalencia e intervalos de confianza al 95\%, usamos el módulo para muestras complejas del Software SPSS (v22). Resultados: Obtuvimos 5.069 registros, edad promedio: $48 \pm 18$ años, $60 \%$ mujeres. Una submuestra de 4.234 cumplió los criterios de la RPC. La muestra expandida (representando 11.279 .865 personas) arrojó los siguientes resultados: riesgo Bajo 60,7\% (CI 95\%, 58-63,4), Medio 31,1\% (28,7-33,6) y Alto 8,2\% (7-9,5). Riesgo en hombres: Bajo

\footnotetext{
* Unidad de Medicina del Sueño, Servicio de Medicina Respiratoria, Instituto Nacional del Tórax.

** Departamento de Estadísticas y Ciencia de la Computación, Universidad de Santiago.

***Departamento de Estudios y Desarrollo, Superintendencia de Salud.
} 
45,8\% (41,7-49,9), Medio 41,1\% (37,3-45,1), Alto 13,1\% (11-15,5). Riesgo en mujeres: Bajo 74,6\% (71,6-77,4), Medio 21,8\% (19,4-24,4), Alto 3,6\% (2,5-5,1). Observamos un incremento del riesgo Alto de AOS desde 0,3\% (0-1,8) en el grupo etario de 18-24 años, a 22,9\% $(18,4-28,2)$ en las personas mayores de 65 años. Los hombres con la mayor prevalencia de Alto riesgo de AOS provenían de 7 de las 15 Regiones de Chile: Araucanía (24\%), Aysén (21,3\%), Coquimbo (18\%), Maule (17,8\%), BioBío (17\%), Arica (16,2\%) y O’Higgins (15,7\%). Conclusiones: La AOS es una condición prevalente en la población chilena, es mayor en hombres que en mujeres y se observó que el riesgo Alto tiende a aumentar con la edad.

Palabras clave: Apnea obstructiva del sueño, riesgo, prevalencia, encuestas y cuestionarios.

\section{Introducción}

La apnea obstructiva del sueño (AOS) es una enfermedad respiratoria crónica caracterizada por un aumento de la resistencia en las vías aéreas superiores, lo cual genera obstrucción total (apneas) o parcial (hipopneas) en forma repetitiva durante el sueño ${ }^{1}$. Son estas obstrucciones repetitivas las que desencadenan los mecanismos fisiopatológicos que tendrán repercusión patológica a corto, mediano y largo plazo, a saber: 1) Cambios en la presión negativa intratorácica; 2) Hipoxia intermitente crónica; 3) Fragmentación del sueño y 4) Privación parcial del sueño ${ }^{1}$.

Entre las consecuencias de la AOS destacan su asociación conocida con: aumento de la morbilidad y mortalidad cardiovascular ${ }^{2,3}$, aumento de trastornos depresivos ${ }^{4-7}$, aumento del riesgo de accidentes de tráfico ${ }^{8-10}$, pérdida de productividad (incluyendo ausentismo laboral) $)^{11-13}$ y deterioro neurocognitivo ${ }^{14,15}$ y de la calidad de vida ${ }^{16-18}$. Todo lo anterior implica un alto costo sanitario, especialmente en relación con los pacientes no $\operatorname{tratados}^{16,19}$.

Los principales factores de riesgo de AOS son la obstrucción de las vías aéreas superiores ${ }^{20,21}$, sexo masculino, el exceso de peso ${ }^{21-23}$, el aumento de la edad ${ }^{21,24}$, la menopausia en mujeres ${ }^{25}$. Otros factores de riesgo son el consumo de medicamentos hipnóticos sedantes ${ }^{26}$ y también sustancias como el alcohol y el tabaco ${ }^{27}$.

El grupo de trabajo para AOS de la American Academy of Sleep Medicine (AASM) propuso en el año 2009, los siguientes criterios de clasificación de gravedad basado en el Índice de Apnea/ hipopnea (IAH). Leve $=$ IAH $\geq 5 \mathrm{y}<15$ eventos/ hora, Moderada $=\mathrm{IAH} \geq 15 \mathrm{y}<30$ eventos/hora y Severa $=I A H \geq 30$ eventos/hora ${ }^{28}$.

Los estudios epidemiológicos muestran que, en los últimos 25 años, la prevalencia de AOS ha estado incrementando en los países occidentales, en paralelo con el aumento de la epidemia de obesidad. Así, por ejemplo, para la AOS moderada/severa, el estudio de la cohorte de Wisconsin
(Estados Unidos) en 1993, mostró una prevalencia de $9 \%$ para los hombres y $4 \%$ para las mujeres ${ }^{29}$. Posteriormente, el estudio de la cohorte de Vitoria-Gasteiz (España) publicado en 2001, mostró una prevalencia de $14 \%$ en hombres y de $7 \%$ en mujeres ${ }^{30}$. El estudio epidemiológico de Sao Paulo (Brasil) en 2010 mostró una prevalencia de $24,8 \%$ en hombres y de $9,6 \%$ en mujeres ${ }^{31}$. El estudio de la cohorte de Lausana (Suiza) en 2015 , encontró una prevalencia de $49,7 \%$ en hombres y de $23,4 \%$ en mujeres ${ }^{32}$. El estudio de Sao Paulo mostró una asociación mucho más alta con el sexo masculino, la obesidad, y la menopausia en las mujeres, y el riesgo aumentaba con la edad en ambos sexos. En este mismo estudio, el nivel socioeconómico bajo mostró ser un factor protector para los hombres, pero mostró ser un factor de riesgo para las mujeres ${ }^{31}$.

Por otra parte, en un estudio de cuatro ciudades latinoamericanas (Ciudad de México, Montevideo, Santiago y Caracas), la población adulta de la ciudad de Santiago tenía una prevalencia de ronquido habitual de $66,4 \%$, somnolencia excesiva diurna de $22,7 \%$ y de la asociación de ronquido + somnolencia + apneas (observadas) de $6,4 \%$, ronquido + apneas de $11 \%$, insomnio de $41,6 \%$, uso de sedantes de $23,7 \%$ y siesta diurna de $28,7 \%$. En todos los síntomas respiratorios del sueño estudiados, en sus distintas asociaciones, así como en insomnio, uso de sedantes y siesta diurna, la prevalencia en la población de Santiago era mucho más elevada que en el resto de las ciudades estudiadas ${ }^{33}$.

Debido al alto costo y la escasa cobertura de la polisomnografía (PSG) -que es el Gold Estándar para el diagnóstico- se han desarrollado dispositivos para diagnóstico ambulatorio y diversos cuestionarios de sueño y reglas de predicción clínica (RPC), con el propósito de contar con herramientas que permitan el tamizaje en población general y en poblaciones con comorbilidades asociadas. Uno de estos, el Cuestionario STOP-Bang (CSB), originalmente desarrollado como herramienta para detectar AOS en pacientes obesos 
candidatos a cirugía bariátrica ${ }^{34}$, posteriormente ha sido aplicado a otras poblaciones quirúrgicas y de pacientes derivados a clínicas de sueño ${ }^{35}$. Es una herramienta de tamizaje replicable, concisa y fácil de usar. Está constituido por ocho preguntas basadas en aspectos clínicos de la AOS (presencia de ronquido fuerte, fatiga/somnolencia diurna, apneas observadas, antecedente de hipertensión arterial, índice de masa corporal (IMC) $>35 \mathrm{~kg} /$ $\mathrm{m}^{2}$, circunferencia de cuello $\geq 43 \mathrm{~cm}$ en hombres $\mathrm{y} \geq 41 \mathrm{~cm}$ en mujeres, sexo masculino), con respuestas dicotómicas (Sí/No) con un puntaje total que va de 0 a 8 . Ha mostrado ser superior que la Escala de Somnolencia de Epworth, el Cuestionario Berlín y el Cuestionario STOP en el tamizaje de pacientes con sospecha de $\operatorname{AOS}^{36}$.

Con el CSB los pacientes pueden ser clasificados por riesgo de AOS basado en los respectivos puntajes. La sensibilidad del puntaje $\geq 3$ para detectar AOS moderada a severa (IAH $\geq 15$ eventos/hora) y AOS severa (IAH $\geq 30$ eventos/ hora) es de $93 \%$ y $100 \%$, respectivamente. Los correspondientes valores predictivos negativos son $90 \%$ y $100 \%$. A medida que el puntaje aumenta desde 0 a 2 hasta 7 a 8 , la probabilidad de AOS moderada a severa aumenta de 18 a $60 \%$ y la probabilidad de AOS severa se eleva de $4 \%$ a $38 \%$. Los pacientes con puntaje en el CSB de 0 a 2 pueden ser clasificados como de bajo riesgo para AOS moderada a severa, mientras que aquellos con puntaje de 5 a 8 pueden ser clasificados como de alto riesgo para AOS moderada a severa. En pacientes cuyos puntajes en el CSB están en el rango medio ( 3 o 4 ), se requieren criterios adicionales para la clasificación. Por ejemplo, un puntaje $\geq 2$ más un $\mathrm{IMC}>35 \mathrm{~kg} / \mathrm{m}^{2}$ podría clasificar a ese paciente como de alto riesgo para AOS moderada a severa. De esta manera, los pacientes pueden ser estratificados por riesgo de AOS de acuerdo con el puntaje en el $\mathrm{CSB}^{37}$.

\section{Pacientes y Método}

El marco muestral de la Encuesta Nacional de Salud 2009-2010 (ENS) fue constituido a partir del Censo de Población y Vivienda del año 2002, excluyendo la II Región de Antofagasta rural y el área urbana de la comuna de Putre, por razones técnicas (porque no se alcanzaba a llegar a un recinto de salud en ninguna de esas dos localidades $^{38}$. La muestra de la ENS 2009-2010 tiene representatividad nacional y regional, dado que las características del diseño muestral (estratificado y multietápico) consideraron las variables región y área urbano/rural, las que, tanto por su tamaño como por sus características, permiten lograr representatividad regional ${ }^{38}$.

El diseño del estudio fue transversal, con una muestra aleatoria de hogares de tipo complejo (estratificada y multietápica por conglomerados) con representatividad nacional, regional y por zona rural/urbana. La población objetivo fueron los adultos de edad mayor o igual a 15 años. La encuesta tuvo una tasa de respuesta en la población elegible de $85 \%$, con una tasa de rechazo de $12 \%$. Finalmente, se entrevistó a 5.434 personas. Una enfermera realizó mediciones clínicas y exámenes a 5.043 participantes y 4.956 aceptaron la realización de exámenes de laboratorio (sangre y orina). La pérdida muestral total de la muestra sobredimensionada fue de $28 \%$ (esto incluye rechazo, no contacto y otras causales de pérdida aleatoria $)^{38}$.

\section{Construcción de la regla de predicción clínica (RPC)}

Como parte de la ENS 2009-2010 en el Formulario 1 , se incluyeron los datos de fecha de nacimiento y sexo, que permitieron construir las variables edad $>50$ años y género masculino. En el mismo formulario se aplicó el Cuestionario del Proyecto PLATINO abreviado sobre trastornos del sueño, el cual fue incluido en el Módulo XIV: Encuesta de 'Síntomas de Sueño'. Este incluía 10 preguntas, de las cuales se utilizaron las respuestas de las tres primeras: 1) ¿Le han dicho que ronca todas o casi todas las noches? (Sí, No, No sabe); 2) ¿Le han dicho que cuando duerme deja de respirar por momentos? (Sí, No, No sabe) y 3) ¿Le cuesta trabajo mantenerse despierto durante el día, por lo menos tres días a la semana? (Sí, No). Las respuestas fueron ajustadas al formato dicotómico Sí/No del CSB y la respuesta 'No sabe' fue computada como perdida por sistema (missing). Con los cuestionarios de autorreporte de diagnósticos realizados por médico se exploró el diagnóstico médico de 25 patologías, entre ellas la hipertensión arterial, y de la situación de tratamiento. También se incluyeron mediciones clínicas (biofisiológicas): antropometría (peso, talla, circunferencia de cintura y cuello), y medición de presión arterial, con tres tomas en forma estandarizada. De esta manera se obtuvo la información de aquellos con diagnóstico previo de hipertensión arterial con y sin tratamiento médico, y de aquellos que presentaron presión arterial elevada al momento de la evaluación en el desarrollo de la ENS 2009-2010, integrándose ambos en la variable "Sospecha de hipertensión arterial", la cual se utilizó como variable de la RPC. 
Con los datos de las mediciones antropométricas se obtuvo el índice de masa corporal (IMC) y la circunferencia de cuello de los sujetos encuestados, lo que permitió incorporar las variables IMC $>35 \mathrm{~kg} / \mathrm{m}^{2}$, y la circunferencia de cuello $\geq 43 \mathrm{~cm}$ en los hombres $\mathrm{y} \geq 41 \mathrm{~cm}$ en las mujeres.

Una vez construidas las variables de la RPC basada en el CSB, se procedió a la suma de los puntajes y a la clasificación en riesgo Bajo $(<3$ puntos), riesgo Medio (3-4 puntos) y riesgo Alto ( $\geq 5$ puntos).

\section{Análisis estadístico}

Se procedió a seleccionar los registros de los sujetos $\geq 18$ años de edad y se realizaron los cálculos de estimación de población, prevalencia, error estándar, intervalo de confianza al 95\% y considerando el efecto de diseño para las variables sexo, riesgo de AOS, grupo etario, estado nutricional, nivel educacional, zona y región de residencia. Se evaluó la existencia de diferencias en la prevalencia en el riesgo de AOS por grupo etario, sexo, región, estado nutricional y nivel de educación usando Chi cuadrado de Pearson considerando el diseño de la encuesta. Para los análisis se utilizó el programa estadístico SPSS versión 22 (IBM Corp.) y el Módulo para muestras complejas del mismo.

\section{Resultados}

Los resultados con la prevalencia de las variables de la RPC se pueden ver en la Tabla 1 y los valores estadísticos de la muestra y las estimaciones poblacionales en la Tabla 2.

La muestra total analizada estaba compuesta por 5.069 sujetos, de los cuales 2.029 (40\%) eran hombres, y $3.040(60 \%)$ eran mujeres. De estos, cumplieron con los requisitos de la RPC un total de 4.234 sujetos, de los cuales 2.496 (59\%) eran de riesgo Bajo, 1.378 (32,5\%) de riesgo Medio, y $360(8,5 \%)$ de riesgo Alto. Hubo una pérdida de 835 registros, correspondiente a $16,5 \%$ de la muestra total, cuyos sujetos no tenían los atributos necesarios para ser clasificados en alguno de los niveles de riesgo de AOS. Para una población estimada de 11.279.865 personas, la población con riesgo Bajo fue de 6.848 .911 , lo que equivale a una prevalencia de $60,7 \%$ (IC 95\%, 58,0- 63,4); la población con riesgo Medio fue de 3.508.753, con una prevalencia de $31,1 \%(28,7-33,6)$; y la población con riesgo Alto fue de 922.201, y una prevalencia estimada de $8,2 \%(7,0-9,5)$. Los resultados con las prevalencias estimadas según sexo y por grupo etario, estado nutricional, nivel educacional, zona y región de residencia se pueden ver en la Tabla 3 .

Tabla 1. Población y prevalencia estimada para las variables de la Regla de Predicción Clínica

\begin{tabular}{|c|c|c|c|c|c|c|}
\hline \multirow[t]{2}{*}{ Variables } & \multicolumn{2}{|c|}{ Hombres } & \multicolumn{2}{|c|}{ Mujeres } & \multicolumn{2}{|c|}{ Total } \\
\hline & Población & $\begin{array}{c}\% \\
\text { (IC al 95\%) }\end{array}$ & Población & $\begin{array}{c}\% \\
\text { (IC al 95\%) }\end{array}$ & Población & $\begin{array}{c}\% \\
\text { (IC al 95\%) }\end{array}$ \\
\hline 1) Ronquido habitual & 3.975 .542 & $\begin{array}{c}68,5 \\
(64,7-72,1)\end{array}$ & 3.327 .692 & $\begin{array}{c}53,9 \\
(50,9-56,8)\end{array}$ & 7.303.234 & $\begin{array}{c}61,0 \\
(58,5-63,3)\end{array}$ \\
\hline 2) Somnolencia diurna & 908.334 & $\begin{array}{c}15,3 \\
(12,5-18,7)\end{array}$ & 1.325 .938 & $\begin{array}{c}20,7 \\
(18,4-23,3)\end{array}$ & 2.234 .273 & $\begin{array}{c}18,1 \\
(16,4-20,1)\end{array}$ \\
\hline 3) Pausas respiratorias & 1.344 .965 & $\begin{array}{c}23,8 \\
(20,8-27,2)\end{array}$ & 817.384 & $\begin{array}{c}13,5 \\
(11,5-15,7)\end{array}$ & 2.162 .349 & $\begin{array}{c}18,5 \\
(16,6-20,5)\end{array}$ \\
\hline 4) Presión arterial elevada & 1.775 .179 & $\begin{array}{c}30,2 \\
(27,0-33,7)\end{array}$ & 1.676 .767 & $\begin{array}{c}26,3 \\
(23,5-29,2)\end{array}$ & 3.451 .946 & $\begin{array}{c}28,2 \\
(26,1-30,3)\end{array}$ \\
\hline 5) IMC $>35 \mathrm{~kg} / \mathrm{m}^{2}$ & 241.719 & $\begin{array}{c}4,2 \\
(3,2-5,4)\end{array}$ & 706.909 & $\begin{array}{c}11,2 \\
(8,8-14,1)\end{array}$ & 948.628 & $\begin{array}{c}7,8 \\
(6,4-9,5)\end{array}$ \\
\hline 6) Edad $>50$ años & 1.816 .128 & $\begin{array}{c}30,7 \\
(27,5-34,0)\end{array}$ & 2.103 .143 & $\begin{array}{c}32,8 \\
(30,0-35,8)\end{array}$ & 3.919 .271 & $\begin{array}{c}31,8 \\
(29,4-34,3)\end{array}$ \\
\hline 7) Circunferencia de cuello* & 778.503 & $\begin{array}{c}13,2 \\
(11,3-15,3)\end{array}$ & 346.797 & $\begin{array}{c}5,4 \\
(4,1-7,2)\end{array}$ & 1.125 .300 & $\begin{array}{c}9,1 \\
(7,9-10,5)\end{array}$ \\
\hline 8) Sexo: Hombre & 5.921 .430 & $\begin{array}{c}100,0 \\
(100,0)\end{array}$ & --------- & --------- & 5.921 .430 & $\begin{array}{c}48,0 \\
(45,5-50,6)\end{array}$ \\
\hline
\end{tabular}

IMC: Índice de masa corporal. *Hombres $\geq 43 \mathrm{~cm}$, Mujeres $\geq 41 \mathrm{~cm}$. 
Tabla 2. Valores estadísticos en la muestra y estimación de población y porcentaje por sexo, riesgo de AOS, edad, IMC, nivel educacional, zona y región de residencia

\begin{tabular}{|c|c|c|c|c|}
\hline \multirow[t]{2}{*}{ Variables } & \multicolumn{2}{|c|}{ Muestra } & \multicolumn{2}{|c|}{ Estimación } \\
\hline & Frecuencia & $\%$ Válido & Población & $\%($ IC $95 \%)$ \\
\hline \multicolumn{5}{|l|}{ Sexo } \\
\hline Hombres & 2.029 & 40,0 & 5.921 .430 & $48,0(45,5-50,6)$ \\
\hline Mujeres & 3.040 & 60,0 & 6.404 .048 & $52,0(49,4-54,5)$ \\
\hline Total & 5.069 & 100,0 & 12.325 .478 & 100,0 \\
\hline \multicolumn{5}{|l|}{ Riesgo de AOS } \\
\hline Bajo $(<3)$ & 2.496 & 59,0 & 6.848 .911 & $60,7(58,0-63,4)$ \\
\hline Medio (3-4) & 1.378 & 32,5 & 3.508 .753 & $31,1(28,7-33,6)$ \\
\hline Alto $(\geq 5)$ & 360 & 8,5 & 922.201 & $8,2(7,0-9,5)$ \\
\hline Total & 4.234 & 100,0 & 11.279 .865 & 100,0 \\
\hline \multicolumn{5}{|l|}{ Grupo etario } \\
\hline 18-24 años & 579 & 11,4 & 2.060 .196 & $16,7(14,7-19,0)$ \\
\hline 25-44 años & 1.734 & 34,2 & 4.951 .410 & $40,2(37,7-42,7)$ \\
\hline 45-64 años & 1.743 & 34,4 & 3.753 .304 & $30,5(27,8-33,2)$ \\
\hline 65 y más & 1.013 & 20,0 & 1.560 .569 & $12,7(11,1-14,4)$ \\
\hline \multicolumn{5}{|l|}{ Estado nutricional } \\
\hline Normal & 1.270 & 27,6 & 3.859 .896 & $31,9(29,3-34,6)$ \\
\hline Sobrepeso & 1.891 & 41,1 & 4.935 .363 & $40,7(38,3-43,2)$ \\
\hline Obesidad & 1.253 & 27,2 & 2.897 .734 & $23,9(21,7-26,3)$ \\
\hline Obesidad mórbida & 128 & 2,8 & 289.129 & $2,4(1,8-3,2)$ \\
\hline \multicolumn{5}{|l|}{ Nivel educacional } \\
\hline Alto (> 12 años) & 980 & 19,4 & 3.197 .580 & $26,0(23,1-29,1)$ \\
\hline Medio (8-12 años) & 2.672 & 52,9 & 6.725 .185 & $54,6(51,6-57,6)$ \\
\hline Bajo (< 8 años) & 1.397 & 27,7 & 2.388 .822 & $19,4(17,6-21,3)$ \\
\hline \multicolumn{5}{|l|}{ Zona } \\
\hline Urbano & 4.305 & 84,9 & 10.734 .804 & $87,1(85,7-88,4)$ \\
\hline Rural & 763 & 15,1 & 1.590 .674 & $12,9(11,6-14,3)$ \\
\hline \multicolumn{5}{|l|}{ Región } \\
\hline I. Tarapacá & 301 & 5,9 & 168.217 & $1,4(1,1-1,7)$ \\
\hline II. Antofagasta & 291 & 5,7 & 365.664 & $3,0(2,7-3,3)$ \\
\hline III. Atacama & 294 & 5,8 & 229.734 & $1,9(1,7-2,1)$ \\
\hline IV. Coquimbo & 292 & 5,8 & 524.292 & $4,3(3,8-4,7)$ \\
\hline V. Valparaíso & 334 & 6,6 & 1.282 .737 & $10,4(8,7-12,3)$ \\
\hline VI. L. Bdo. O’Higgins & 299 & 5,9 & 620.458 & $5,0(4,3-5,8)$ \\
\hline VII. Maule & 337 & 6,6 & 734.300 & $6,0(5,1-6,9)$ \\
\hline VIII. Biobío & 276 & 5,4 & 1.461 .685 & $11,9(9,7-14,5)$ \\
\hline IX. La Araucanía & 318 & 6,3 & 728.402 & $5,9(5,0-7,0)$ \\
\hline X. Los Lagos & 308 & 6,1 & 536.187 & $4,4(3,7-5,1)$ \\
\hline XI. Aysén & 270 & 5,3 & 79.511 & $0,6(0,6-0,7)$ \\
\hline XII. Magallanes y Antártica & 306 & 6,0 & 156.479 & $1,3(1,1-1,5)$ \\
\hline XIII. R. Metropolitana & 867 & 17,1 & 5.038 .440 & $40,9(38,0-43,9)$ \\
\hline XIV. Los Ríos & 287 & 5,7 & 247.916 & $2,0(1,7-2,4)$ \\
\hline XV. Arica y Parinacota & 289 & 5,7 & 151.455 & $1,2(1,1-1,4)$ \\
\hline Total & 5.069 & 100,0 & 12.325 .478 & 100,0 \\
\hline
\end{tabular}




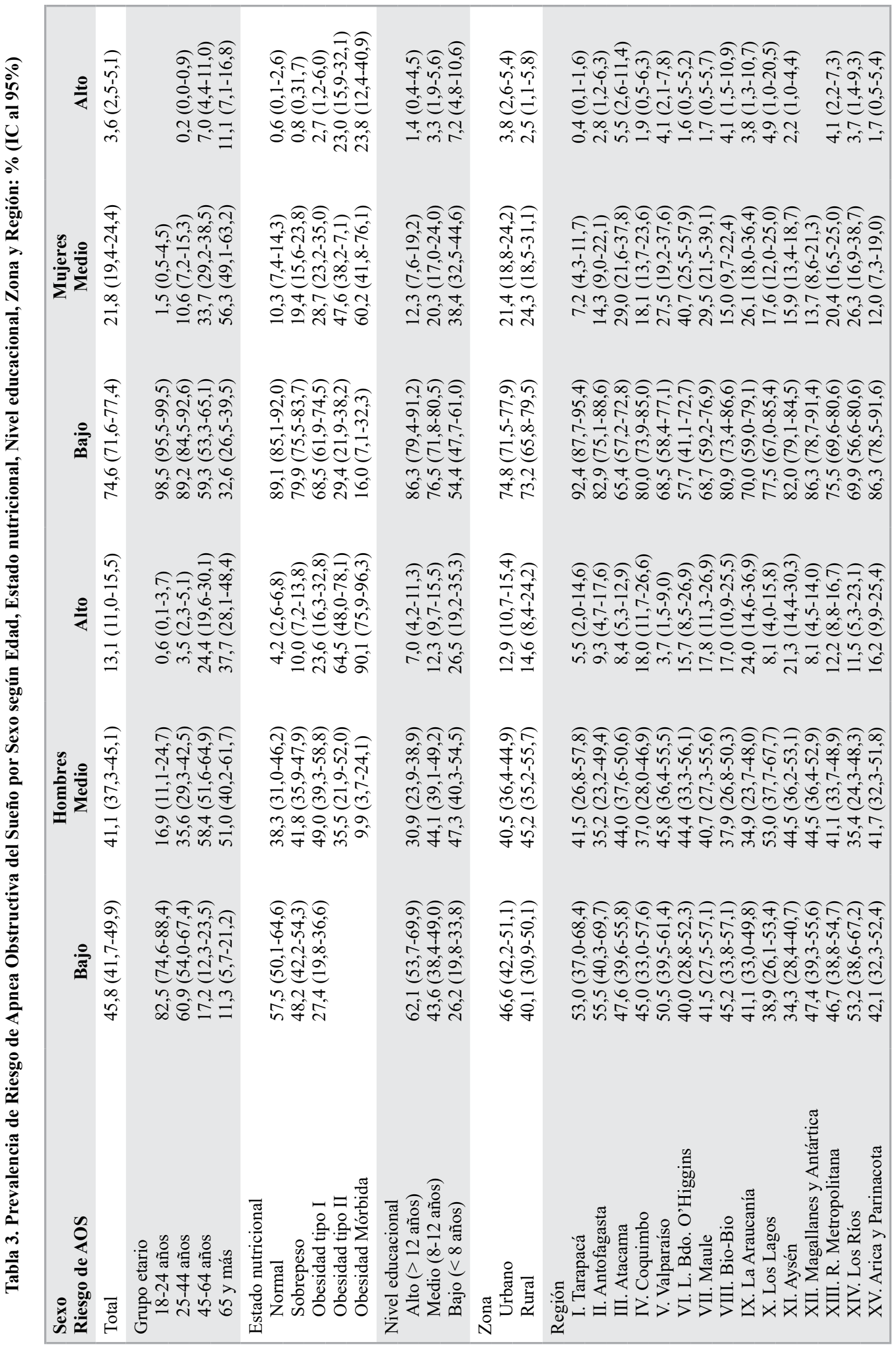




\section{Discusión}

Nuestro estudio muestra una alta prevalencia de riesgo alto y medio de AOS especialmente en hombres y población de mayor edad. Entre las fortalezas del estudio está el hecho de que se basa en una muestra de la población adulta de Chile y que tiene representatividad regional.

Una debilidad potencial del estudio es la estimación del riesgo de AOS mediante una metodología que no incorpora estudios de diagnóstico del sueño mediante polisomnografía u otro método validado. Sin embargo, los estudios publicados han validado el CSB como una herramienta para estimar riesgo de AOS de grado moderado a severo. Esto es importante, al menos por tres motivos: 1) porque permite realizar confirmación diagnóstica en una segunda etapa con métodos abreviados y ambulatorios que no requieren de una polisomnografía, dada la existencia de una alta probabilidad pre-test; 2) porque la mayoría de los estudios y guías clínicas recomiendan tratar con presión positiva a los pacientes con IAH $>15$ eventos/hora, que es el grupo más afectado por las consecuencias de la enfermedad, y el que más se beneficia con dicha terapia y 3 ) porque los pacientes con AOS leve, e incluso muchos con un grado moderado, pueden beneficiarse con tratamientos diferentes y menos costosos.

Dadas las diversas consecuencias que la AOS tiene para la salud, la estimación de su prevalencia es importante para el diseño de políticas de salud, programas de prevención primaria y secundaria, focalización en el manejo de las diversas comorbilidades asociadas, y también para el rediseño curricular en la formación de los profesionales de la salud.

Las implicancias económicas y para la salud, de acuerdo a estimaciones internacionales, que podrían medirse con algún método cuantitativo más elaborado, son dos puntos abiertos que no se pueden abordar por ahora, quedando pendiente para estudios posteriores. No obstante, es importante señalar que diversos estudios publicados han mostrado que el tratamiento de la AOS es altamente costo-efectivo, tanto para los pacientes y sus familias, como para el sistema sanitario ${ }^{19,39,40}$.

\section{Conclusiones}

Según los resultados de nuestro estudio, la prevalencia de riesgo Medio y Alto de apnea obstructiva del sueño moderada a severa es elevada en la población chilena adulta. Esta prevalencia es mayor en hombres que en mujeres y aumenta con la edad y con el exceso de peso. Se relaciona de manera inversa con el nivel educacional. Es preocupante el hallazgo de nueve regiones en que la prevalencia de riesgo Alto en hombres supera el $10 \%$, en siete de ellas supera el $15 \%$ y en dos supera el $20 \%$, respectivamente.

\section{Bibliografía}

1.- PATIL SP, SCHNEIDER H, SCHWARTZ AR, SMITH PL. Adult obstructive sleep apnea: pathophysiology and diagnosis. Chest 2007; 132: 325-37.

2.- BAUTERS F, RIETZSCHEL ER, HERTEGONNE KB, CHIRINOS JA. The Link Between Obstructive Sleep Apnea and Cardiovascular Disease. Curr Atheroscler Rep. 2016;18:1. doi: 10.1007/s11883-015-0556-z.

3.- BRADLEY TD, FLORAS JS. Obstructive sleep apnoea and its cardiovascular consequences. Lancet 2009; 373 (9657): 82-93.

4.- LU MK, TAN HP, TSAI IN, HUANG LC, LIAO XM, LIN SH. Sleep apnea is associated with an increased risk of mood disorders: a population-based cohort study. Sleep Breath. 2017; 21:243-53. doi: 10.1007/s11325016-1389-x

5.- KERNER NA, ROOSE SP. Obstructive Sleep Apnea is Linked to Depression and Cognitive Impairment: Evidence and Potential Mechanisms 2016; 24496-508.

6.- EDWARDS C, MUKHERJEE S, SIMPSON L, PALMER LJ, ALMEIDA OP, HILLMAN DR. Depressive Symptoms before and after Treatment of Obstructive Sleep Apnea in Men and Women. Journal of clinical sleep medicine: 2015; 11: 1029-38.

7.- SCHRODER CM, O'HARA R. Depression and Obstructive Sleep Apnea (OSA). Annals of general psychiatry. 2005;4:13. doi:10.1186/1744-859X-4-13.

8.- TREGEAR S, RESTON J, SCHOELLES K, PHILLIPS B. Obstructive sleep apnea and risk of motor vehicle crash: systematic review and meta-analysis. Journal of clinical sleep medicine 2009; 5: 573-81.

9.- RODENSTEIN D. Sleep apnea: traffic and occupational accidents-individual risks, socioeconomic and legal implications. Respiration 2009; 78: 241-8.

10.- KARIMI M, HEDNER J, HABEL H, NERMAN O, GROTE L. Sleep apnea-related risk of motor vehicle accidents is reduced by continuous positive airway pressure: Swedish Traffic Accident Registry data. Sleep 2015; 38: 341-9.

11.- EGEA SANTAOLALLA CJ, DEL CAMPO MATIAS F. Work-related Accidents, Absenteeism and Productivity in Patients With Sleep Apnea. A Future Consideration in Occupational Health Assessments? Arch bronconeumol 2015; 51: 209-10.

12.- GUGLIELMI O, JURADO-GAMEZ B, GUDE F, BUELA-CASAL G. Occupational health of patients 
with obstructive sleep apnea syndrome: a systematic review. Sleep Breath 2015; 19: 35-44.

13.- JURADO-GAMEZ B, GUGLIELMI O, GUDE F, BUELA-CASAL G. Workplace accidents, absenteeism and productivity in patients with sleep apnea. Arch bronconeumol 2015; 51: 213-8.

14.- LAL C, STRANGE C, BACHMAN D. Neurocognitive impairment in obstructive sleep apnea. Chest 2012; 141: 1601-10.

15.- RAMOS AR, TARRAF W, RUNDEK T, REDLINE S, WOHLGEMUTH WK, LOREDO JS, et al. Obstructive sleep apnea and neurocognitive function in a Hispanic/ Latino population. Neurology 2015; 84: 391-8.

16.- AURORA RN, QUAN SF. Quality Measure for Screening for Adult Obstructive Sleep Apnea by Primary Care Physicians. Journal of clinical sleep medicine 2016; 12 (8): 1185-7.

17.- COMAN AC, BORZAN C, VESA CS, TODEA DA. Obstructive sleep apnea syndrome and the quality of life. Clujul Med 2016; 89: 390-5.

18.- LEE W, LEE SA, RYU HU, CHUNG YS, KIM WS. Quality of life in patients with obstructive sleep apnea: Relationship with daytime sleepiness, sleep quality, depression, and apnea severity. Chronic respiratory disease 2016; 13: 33-9.

19.- POUllie AI, COGNeT M, GAUTHIER A, CLEMENTZ M, DRUAIS S, SPATH HM, et al. CostEffectiveness of Treatments for Mild-to-Moderate Obstructive Sleep Apnea in France. Int J Technol Assess Health Care 2016; 32: 37-45.

20.- WILHELM CP, DESHAZO RD, TAMANNA S, ULLAH MI, SKIPWORTH LB. The nose, upper airway, and obstructive sleep apnea. Annals of allergy, asthma \& immunology 2015; 115: 96-102.

21.- YOUNG T, SKATRUD J, PEPPARD PE. Risk factors for obstructive sleep apnea in adults. JAMA 2004; 291 : 2013-6.

22.- YILDIRIM Y, YILMAZ S, GUVEN M, KILINC F, KARA AV, YILMAZ Z, et al. Evaluation of Anthropometric and Metabolic Parameters in Obstructive Sleep Apnea. Pulmonary medicine. 2015;2015:189761. doi: 10.1155/2015/189761.

23.- PLEAVA R, GAITA D, ARDELEANU C, FRENTZ $\mathrm{S}$, UDRESCU M, UDRESCU L, et al. Obesity in association with Sleep Apnea Syndrome as predictor for coronary-vascular comorbidities. Pneumologia 2016; 65: 14-8.

24.- HONGYO K, ITO N, YAMAMOTO K, YASUNOBE Y, TAKEDA M, OGURO R, et al. Factors associated with the severity of obstructive sleep apnea in older adults. Geriatr Gerontol Int 2017; 17: 614-21.

25.- TAVARES E CASTRO A, DUARTE JC, CRAVO J, FREITAS S, MATOS MJ. Obstructive sleep apnea in women: Prevalence, risk factors and relation to menopausal status. Rev Port Pneumol 2014; 20: 342-3.

26.- JIRAPINYO P, THOMPSON CC. Sedation Challenges:
Obesity and Sleep Apnea. Gastrointestinal endoscopy clinics of North America 2016; 26: 527-37.

27.- LUI MM, MAK JC, LAI AY, HUI CK, LAM JC, LAM DC, et al. The Impact of Obstructive Sleep Apnea and Tobacco Smoking on Endothelial Function. Respiration 2016; 91: 124-31.

28.- EPSTEIN LJ, KRISTO D, STROLLO PJ, JR., FRIEDMAN N, MALHOTRA A, PATIL SP, et al. Clinical guideline for the evaluation, management and long-term care of obstructive sleep apnea in adults. Journal of clinical sleep medicine 2009; 5: 263-76.

29.- YOUNG T, PEPPARD PE, GOTTLIEB DJ. Epidemiology of obstructive sleep apnea: a population health perspective. Am J Respir Crit Care Med 2002; 165 : 1217-39.

30.- DURÁN J, ESNAOLA S, RUBIO R, IZTUETA A. Obstructive sleep apnea-hypopnea and related clinical features in a population-based sample of subjects aged 30 to 70 yr. Am J Respir Crit Care Med 2001; 163 (3 Pt 1): $685-9$.

31.- TUFIK S, SANTOS-SILVA R, TADDEI JA, BITTENCOURT LR. Obstructive sleep apnea syndrome in the Sao Paulo Epidemiologic Sleep Study. Sleep Med 2010; 11: 441-6.

32.- HEINZER R, VAT S, MARQUES-VIDAL P, MARTISOLER H, ANDRIES D, TOBBACK N, et al. Prevalence of sleep-disordered breathing in the general population: the HypnoLaus study. Lancet Respir Med 2015; 3 : 310-8.

33.- BOUSCOULET LT, VAZQUEZ-GARCÍA JC, MUINO A, MÁRQUEZ M, LÓPEZ MV, DE OCA MM, et al. Prevalence of sleep related symptoms in four Latin American cities. Journal of clinical sleep medicine: 2008; 4: 579-85.

34.- CHUNG F, YEGNESWARAN B, LIAO P, CHUNG SA, VAIRAVANATHAN S, ISLAM S, et al. STOP questionnaire: a tool to screen patients for obstructive sleep apnea. Anesthesiology 2008; 108: 812-21.

35.- NAGAPPA M, LIAO P, WONG J, AUCKLEY D, RAMACHANDRAN SK, MEMTSOUDIS S, et al. Validation of the STOP-Bang Questionnaire as a Screening Tool for Obstructive Sleep Apnea among Different Populations: A Systematic Review and Meta-Analysis. PloS one 2015; 10 (12): e0143697.

36.- LUO J, HUANG R, ZHONG X, XIAO Y, ZHOU J. STOP-Bang questionnaire is superior to Epworth sleepiness scales, Berlin questionnaire, and STOP questionnaire in screening obstructive sleep apnea hypopnea syndrome patients. Chinese medical journal 2014; 127 : 3065-70.

37.- CHUNG F, ABDULLAH HR, LIAO P. STOP-Bang Questionnaire: A Practical Approach to Screen for Obstructive Sleep Apnea. Chest 2016; 149: 631-8.

38.- MINSAL. Encuesta Nacional de Salud (ENS) 20092010. Cap. III: Metodología. In: Pública DdS, editor. MINSAL Chile 2010. 
39.- MCMILLAN A, BRATTON DJ, FARIA R, LASKAWIEC-SZKONTER M, GRIFFIN S, DAVIES RJ, et al. A multicentre randomised controlled trial and economic evaluation of continuous positive airway pressure for the treatment of obstructive sleep apnoea syndrome in older people: PREDICT. Health Technol Assess 2015;
19: $1-188$

40.- PIETZSCH JB, GARNER A, CIPRIANO LE, LINEHAN JH. An integrated health-economic analysis of diagnostic and therapeutic strategies in the treatment of moderate-to-severe obstructive sleep apnea. Sleep 2011; 34: 695-709.

Correspondencia a:

Dr. Juan Carrillo Azócar

Unidad de Medicina del Sueño, Servicio de Medicina

Respiratoria, Instituto Nacional del Tórax.

Av. José M. Infante 717, Piso 4, Providencia, Santiago,

Chile.

Email: jcarrillo@torax.cl 